\title{
Plasma Line Measurements at Chatanika With High-Speed Correlator and Filter Bank
}

\author{
WLODEK, KOFMAN ${ }^{1}$ AND VINCENT WICKWAR
}

\author{
Radio Physics Laboratory, SRI International, Menlo Park, California 94025
}

\begin{abstract}
In the spring and fall of 1978 we made an extensive series of plasma line and correlative observations with the Chatanika incoherent scatter radar. To make these measurements, we greatly modified the radar receiving system. In addition to enlarging the plasma line filter bank the most significant change was the incorporation of a high-speed correlator provided by the French. This was the first use of a correlator in a monostatic radar to obtain the internsity spectra of naturally occurring plasma lines. In this paper we develop the signal-processing theory that we use to obtain the plasma line intensities from these measurements; we also show that these intensities compare well with those obtained from the filter bank. To show the richness of the phenomena and to explore the capabilities of the correlator, we examine a wide variety of spectra that have been enhanced by secondary electrons in the auroral $E$ layer. From the other simultaneous measurements we are able to relate these spectra and their variations to the auroral situation. We also obtained the first measurements in the auroral region of photoelectron-excited plasma lines in the $E$ and $F$ layers. Perhaps most significant, in the plasma line spectra we detected a Doppler shift that we then used to determine the Birkeland current carried by ambient electrons. Although there is a large estimated uncertainty for this first determination, we obtained a downward Birkeland current of $10 \mu \mathrm{A} /$ $\mathrm{m}^{2}$ in the diffuse aurora in what is, most likely, the equatorward portion of the evening sector auroral oval.
\end{abstract}

\section{INTRODUCTION}

Most previous measurements made with the Chatanika radar have been of the ion component of the incoherent scatter spectrum. However, it is also possible to observe the electron component of the spectrum, the plasma lines [Wickwar, 1978]. (A general discussion of the incoherent scatter technique and these components of the spectrum has been presented by Evans [1969].) While plasma lines are more difficult to observe, they are important because they are a source, or potential source, of more precise information about physical parameters already accessible with the ion component: electron density and the electron-to-ion temperature ratio. They also provide new information about other physical processes and parameters, such as the interaction of auroral primaries with the neutral atmosphere, low-energy secondary electrons, and the current carried by thermal electrons.

During the spring and fall of 1978 we performed an extensive series of joint American-French plasma line observations at Chatanika. To undertake these plasma line and correlative ion component observations, the data acquisition system and observation program were extensively modified. The plasma line filter bank [Wickwar, 1978] was expanded from 3 to 12 filters, and a high-speed digital correlator from Grenoble [Chabert et al., 1974] was integrated into the system.

The use of a correlator to measure the intensity or temperature spectrum of the naturally occurring plasma line is, alone, a significant advance. The technique differs in significant ways from that used for the ion component [Farley, 1969] or that used for the enhanced plasma line in the ionospheric modification experiments [Carlson et al., 1972; Kantor, 1974]. Furthermore, while a correlator was previously used to determine the variations in the critical frequency [Showen, 1979], the correlator measurements here go beyond those, in that we determined the intensity spectrum.

\footnotetext{
${ }^{1}$ Permanent address is Centre d'Etude des Phénomènes Aléatoires et Géophysiques, BP 46, 38402 St. Martin d'Heres, France.

Copyright (C) 1980 by the American Geophysical Union.
}

In this paper we develop the theory for interpreting the correlator measurements; we compare the resultant intensities with those from the filter bank and relate variations in the spectra to variations in the auroral $E$ layer. In addition, in what could prove to be the most important result, we deduce the Birkeland current carried by ambient or thermal electrons.

\section{RADAR SYSTEM AND MEASUREMENTS}

The location of the radar and the parameters of the radar system have been described in several publications [Leadabrand et al., 1972; Rino, 1972; Rino et al., 1974a; Baron, 1977] and reports [Burch, 1970, 1975; Rino et al., 1974b; Dawson, 1976; Petriceks, 1976]. To make our observations, we modified the system. Principally, this involved decreasing the sample interval from 10 to $5 \mu \mathrm{s}$, increasing the external integrator from 1024 to 2048 words, adding nine plasma line filters, and incorporating the French autocorrelator (FAC) into the system. The resultant receiver system is shown in Figure 1. Except for the branch including the FAC, this is the system currently available for all ionospheric measurements at Chatanika.

To accommodate the changes in the system and the addition of the FAC, the observation program, Atol [Dawson, 1976], had to be extensively modified.

This system and observing program enabled us to make an unusually complete set of measurements. We used three pulses (or set of pulses) at intervals of either 10 or $8.3 \mathrm{~ms}$ to obtain two different sets of power measurements and to sample with three autocorrelators. The experimental setup is described in Table 1 . In addition, we usually switched plasma line receivers at the conclusion of each on-line integration period (2-20 s) so as to have substantially simultaneous measurements of upshifted and downshifted plasma lines.

The procedures for making and interpreting most of these measurements have been described elsewhere. Power measurements of the Chatanika ion component have been described by Leadabrand et al. [1972] and Baron [1974, 1977]. Ion component spectral measurements with the Chatanika 


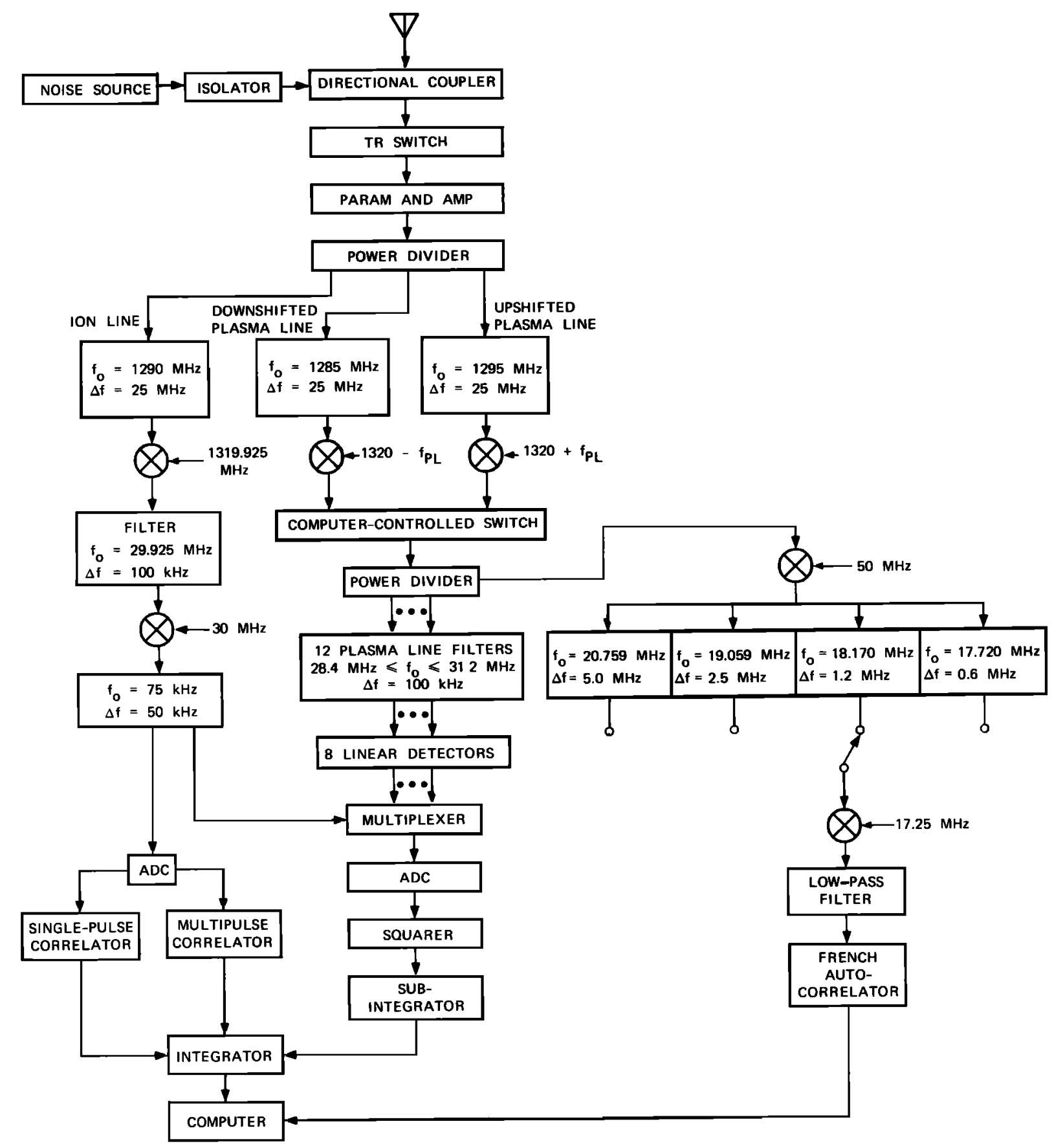

Fig. 1. Block diagram of the analog and digital parts of the receiver system.

single-pulse correlator have been described by Rino [1972]; those with the Chatanika multipulse correlator by Rino et al. [1974a, 1977] and Baron [1977]. Power measurements of plasma lines have been discussed by Yngvesson and Perkins [1968], Wickwar [1971], Cicerone and Bowhill [1971], VidalMadjar et al. [1975], and Wickwar [1978].

Plasma line spectral measurements were made with the FAC [Chabert et al., 1974]. It is a high-speed multibit correlator capable of sampling at rates as high as $15 \mathrm{MHz}$ and producing 256-point correlation functions in real time. The processing starts by filling a delay line with the first 256 samples; thereafter a complete correlation function is produced and stored for each new sample. The sampling continues for a time much longer than the time needed to fill the delay line.
The procedure for interpreting the correlator measurements in terms of the plasma line intensity spectrum is developed in the next section.

To compare plasma line intensities at different frequencies, in particular, upshifted and downshifted plasma lines, it is necessary to have a good gain versus frequency calibration of the entire radar system. To do this, the radio source, Cassiopeia, was observed over a $24-\mathrm{MHz}$ range centered on the transmitter frequency at $1290 \mathrm{MHz}$ (J. D. Kelly, private communication, 1978). The drift curves show no detectable variation with frequency in the shape of the antenna pattern. The received signal strength when corrected for a $1.2 \%$ decrease in the signal from Cassiopeia over the frequency range examined therefore indicates the relative one-way gain of the antenna 
TABLE 1. The Measurements

\begin{tabular}{|c|c|c|c|c|c|c|c|c|}
\hline Measurement & $\begin{array}{c}\text { Pulse } \\
\text { Length, } \\
\text { } \mu \mathrm{S}\end{array}$ & $\begin{array}{c}\text { Range } \\
\text { Resolu- } \\
\text { tion, } \\
\text { km }\end{array}$ & $\begin{array}{c}\text { Useful } \\
\text { Range } \\
\text { Interval, } \\
\text { km }\end{array}$ & $\begin{array}{c}\text { Sampling } \\
\text { Interval or } \\
\text { Frequency, } \\
\quad \mu s\end{array}$ & $\begin{array}{c}\text { Num- } \\
\text { ber } \\
\text { of } \\
\text { Gates }\end{array}$ & $\begin{array}{l}\text { Number of } \\
\text { Samples/ } \\
\text { Gate }\end{array}$ & Lags & $\begin{array}{c}\text { Filter Band- } \\
\text { width of } \\
\text { Equivalent } \\
\text { Filter, } \\
\text { kHz }\end{array}$ \\
\hline $\begin{array}{l}\text { Power (density) } \\
\text { Filter bank (plasma line } \\
\text { intensities) }\end{array}$ & $\begin{array}{r}60 \\
320\end{array}$ & $\begin{array}{c}9 \\
\sim 2^{b}\end{array}$ & $\begin{array}{l}70-720 \\
50-375\end{array}$ & $\begin{array}{l}10 \\
40^{d}\end{array}$ & $\begin{array}{l}600^{e} \\
75^{d . e}\end{array}$ & $\begin{array}{l}1 \\
1\end{array}$ & & $\begin{array}{c}50 \\
100^{\prime}\end{array}$ \\
\hline $\begin{array}{l}\text { SPC (temperatures and Ion } \\
\text { velocities) }\end{array}$ & 320 & $\sim 50^{c}$ & $115-465$ & 10 & $9^{\prime}$ & 32 & 32 & 1.56 \\
\hline $\begin{array}{l}\text { MAC (temperatures, collision } \\
\text { frequencies, and ion velocities) }\end{array}$ & $60^{\circ}$ & 10 & $80-150$ & 10 & $15^{\prime}$ & $33^{h}$ & $32^{h}$ & 1.56 \\
\hline $\begin{array}{l}\text { FAC (plasma line intensity } \\
\text { spectra }\end{array}$ & 320 & $\sim 50^{c}$ & $\begin{array}{c}E \text { and } F \text { region } \\
\text { peaks }\end{array}$ & $\begin{array}{l}15,8,4, \text { or } 2 \\
\mathrm{MHz}\end{array}$ & $4^{g}$ & $\begin{array}{c}4800,2560,1280 \\
\text { or } 640\end{array}$ & 256 & $\begin{array}{l}30,16,8 \text {, } \\
\quad \text { or } 4\end{array}$ \\
\hline \multicolumn{9}{|c|}{ 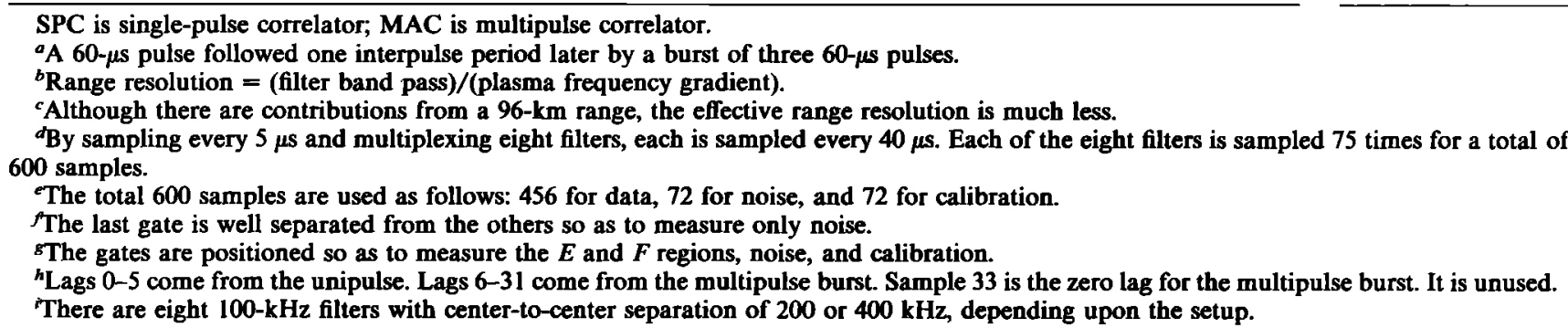 } \\
\hline
\end{tabular}

over this frequency range. This is the appropriate quantity for the plasma line measurements because transmission and reception occur at different frequencies. The resultant relative gain $G(\nu)$ is plotted in Figure 2. Although this calibration is relative to the noise source, separate calibrations of the noise source (by the manufacturer, Microwave Semiconductor Corporation), the isolator, and the directional coupler (J. D. Kelly, private communication, 1978) indicate that these components have only a minor effect upon the calibration. The fully corrected or absolute calibration curve is also shown in Figure 2.

\section{THEORY AND TECHNIQUE}

In this section we discuss the theory that we applied to the plasma line measurements. In the first part our discussion focuses on that portion of the theory that is necessary to relate the FAC-measured autocorrelation functions and deduced spectra to those of the medium. In the second part we show how we determined the plasma line intensity (plasma wave temperature) from the spectral measurements and other ionospheric and radar parameters. In the third part we present the method that we used to look for the velocity of the ambient electrons and hence for the current parallel to the magnetic field.

\section{a. Theory of Plasma Line Correlation Measurements}

The correlation technique has long been used successfully [Evans, 1969] to measure the ion spectrum. The signal-processing theory for these correlation functions has been described by Farley [1969] and Rino [1972]. We have extended that theory to apply to correlation measurements of the plasma line spectrum.

The two signal-processing theories differ significantly from one another. For the ion spectrum we assume that the received spectrum is constant over the scattering volume. This assumption is usually good because we select a sufficiently short pulse length. For the plasma lines this assumption, how- ever, does not hold, since the spectrum at each altitude depends on the density profile. In the first approximation the plasma line spectrum is

$$
S(f)=A(z) \delta[f-f(z)]
$$

where amplitude $\boldsymbol{A}$ and plasma line frequency $f$ are both functions of altitude $z$.

The signal-processing theory for the plasma lines is developed in detail in Appendix A. Here we present the major results. The expected value of the measured autocorrelation function is

$\mathbf{E}\left\{R_{s}\right\} \simeq \frac{1}{N} \sum_{m=-(N-1)}^{N-1}\left(1-\frac{|m|}{N}\right) R_{\Delta N_{e}}\left[n \Delta t ; \frac{c^{\prime}\left(\tau_{r}+m \Delta t\right)}{2}\right]$

where $\boldsymbol{R}_{\overline{\Delta N_{c}}}$ is the autocorrelation function of the medium.

This equation is basic to our results because it relates the measured autocorrelation function to that of the medium. It can be understood physically with the aid of the range-time diagram in Figure 3. At each range within the range gate there

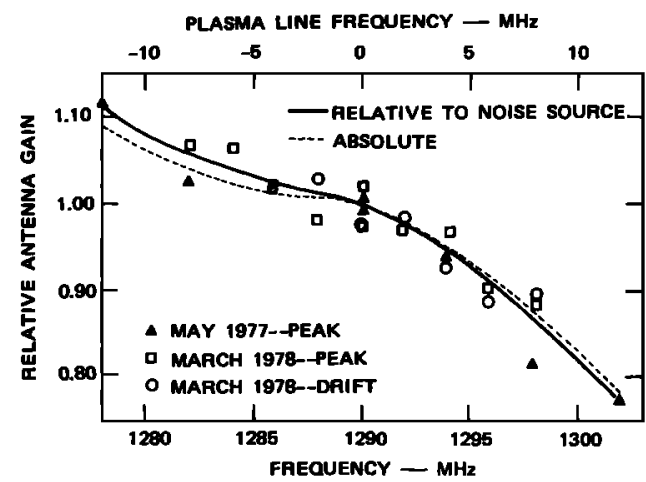

Fig. 2. Antenna calibration. The solid curve indicates the gain $G(\nu)$ relative to the calibration noise source. This is the curve used in the data analysis. By including corrections for the frequency dependence of the noise source, isolator, and directional coupler the dashed curve shows the actual frequency dependence of the antenna receiving system. 
is a different correlation function because the plasma frequency or its gradient is different. The measured correlation function is the sum of these different correlation functions; it is weighted at each range by the number of measurements or duration of measurement. At range $r$, defined by $m=0$, there are $N$ contributions to the measured correlation function obtained over a time period $T$. At range $r^{\prime}$, defined by $0<m^{\prime}<$ $N-1$, there are $N\left(1-m^{\prime} / N\right)=N-m^{\prime}$ contributions to the measured correlation function obtained over a time period $T^{\prime}=T\left(1-m^{\prime} / N\right)$.

Now let us turn from the autocorrelation function to the spectrum. Equation (2) becomes

$\mathbf{E}\left\{\hat{S}_{s}(f)\right\} \cong \frac{1}{N} \sum_{m=-(N-1)}^{N-1}\left(1-\frac{|m|}{N}\right) S_{\Delta N_{r}}\left[f ; \frac{c^{\prime}\left(\tau_{r}+m \Delta t\right)}{2}\right]$

in the frequency domain. This is the composite spectrum from all altitudes. If the correlator gate is positioned at an altitude such that the plasma line frequency varies monotonically within the gate, then the part of the spectrum from a small altitude region is given by

$$
\mathbf{E}\left\{\hat{S}_{s}\left(f_{i}\right)\right\} \cong \frac{N_{i}}{N} S_{\Delta N_{e}}\left[f_{i} ; \frac{c^{\prime}\left(\tau_{r}+m_{P} \Delta t\right)}{2}\right]
$$

where

$$
N_{\imath}=1-\frac{\left|m_{\mathrm{t}}\right|}{N}
$$

and $m_{i}$ is determined from the altitude at which $f$, occurs. We call $N_{1}$ the 'altitude weighting function.' The plasma line spectrum in the medium, $S_{\overline{\Delta N_{e}}}$, can thus be obtained at each altitude within the correlator gate. If the center of the correlator gate is positioned at the peak of the $E$ or $F$ layer, then there could be two contributions to the observed spectrum, one from above and one from below.

We also have to consider the effects of the receiver bandwidth upon the received plasma line signal. The receiver system, up to the point where the correlation function is formed, can be represented by a single filter with the impulse response

$$
h(t) \stackrel{F T(t)}{\leftrightarrows} H(f)
$$

The spectrum at the output of the receiver then becomes

$$
S_{s}(f ; t) \simeq|H(f)|^{2} S_{s}(f ; t)
$$

The influence of the filter is the same as that for the case of stationary signals. Thus the measured spectrum is the product of the filter spectrum and the composite spectrum of the medium, (3). Finally, the relation between the measured spectrum and the spectrum of the medium in a small altitude region is obtained by combining (4) and (5).

\section{b. Determination of $k T_{p}$}

To obtain the plasma wave temperature $k T_{p}$, we apply the radar equation for the power $P_{r}$ received in the electron component [Yngvesson and Perkins, 1968]

$$
P_{r}=k T_{A} B=\frac{P_{T}}{r^{2}} \frac{2 \pi \sigma_{e} k T_{p}}{\lambda^{2} e^{2}} \delta_{r} A\left(\nu, \nu_{0}\right)
$$

where

$k$ Boltzman's constant;

$T_{A}$ antenna temperature;
$B$ bandwidth of the plasma line filter or of the FAC equivalent filter;

$P_{r}$ transmitter peak power;

$r$ range;

$\sigma_{e}$ radar cross section of the electron;

$\lambda$ radar wavelength;

$e$ charge on the electron;

$\delta_{r}$ region in range from which the signal is scattered; $A\left(\nu, \nu_{0}\right)$ effective area of the antenna for a signal transmitted at frequency $\nu_{0}$ and received at $\nu$, equal to $A\left(\nu_{0}\right) G(\nu)$.

This equation may then be solved directly for $k T_{p}$ :

$$
k T_{p}=4.99 \times 10^{-7} \frac{B T_{A} r^{2}}{P_{T} G(\nu) \delta_{r}} \quad \mathrm{eV}
$$

where $A\left(\nu_{0}\right)=172 \mathrm{~m}^{2}$ was found by using the analogous ion component radar equation and electron densities determined from the plasma line frequencies. As in previous applications [Yngvesson and Perkins, 1968; Wickwar, 1971, 1978; Cicerone and Bowhill, 1971], $k T_{p}$ may also be found in an alternate manner, using the ratio of the electron and ion component radar equations. Indeed, the fact that we obtain the same $k T_{p}$ values by the two methods is a good independent verification of $A\left(\nu_{0}\right)$.

To use this equation, we need to employ two other parameters besides the antenna temperature of the plasma line signal. The first is the range of the signal. This is obtained directly for the measurements with the filter bank. Provided that the range is constant during the integration and that the filter is not at the critical frequency, the signal range can be found to within $2 \mathrm{~km}$ on the topside of the auroral $E$ layer [Wickwar, 1978 ] and $5 \mathrm{~km}$ on the topside of the $F$ layer. For the correlator the signal range is found indirectly from either the filter bank or the electron density profile. In the latter case the upper cutoff frequency is associated with the range of the $E$ or $F$ layer critical frequency. The ranges of the other frequencies are then obtained from the fully corrected profile. It is apparent that there could be a range ambiguity due to signals from both the bottomsides and topsides of the layers. A careful placement of the receiver gate, however, will eliminate that ambiguity in the $F$ region except for a small determinable range of frequencies near the critical frequency. In the $E$ region there appears to be no ambiguity problem because the signals from the bottomside are neglibly small. So far, they have been too weak to detect with the filter bank (see Wickwar [1978] and section $4 a$ of this paper). There is no ambiguity between $E$ and $F$ layers because they are quite far apart in comparison to the 48-km range gate. Again, these procedures for finding the signal range work best for a stable $E$ or $F$ layer.

The second parameter is the region $\delta$, from which the radar signal is scattered. For a given plasma line filter passband or for a given width of the FAC equivalent filter this region is calculated from the electron density or plasma frequency gradients. These gradients are determined from the variation in range of the signals from the filter bank or from the electron density profile. In either case we assume that the gradient varies smoothly in range.

\section{c. Electron Velocity and Current Measurements in the Auroral E Layer}

The velocity of the ambient electrons is found from the Doppler shift of the radar signal [Bauer et al., 1976]. It is determined from the difference between the upshifted and 
downshifted plasma line measurements. The current is derived from the electron density and the difference between the electron and ion velocities, all of which are measured simultaneously. Since the measurements have been made along the magnetic field line, they are sensitive to a component of the current not previously measured with the radar.

To find the frequency differences between the upshifted and downshifted spectra, it would be easiest if there were a sharp feature in the spectrum. However, the plasma line spectra vary smoothly and slowly with the possible exception of the cutoff at the high-frequency ends. However, as we shall see, this cutoff may in fact be very gradual in the auroral $E$ layer. We therefore use the fact that the two spectra are symmetric and determine the shifts of the whole spectra from a first-moment calculation

$$
f_{\text {Doppler }}=\frac{f_{\text {up }}+f_{\text {down }}}{2}=\frac{\int_{-\infty}^{\infty} f s(f) d f}{\int_{-\infty}^{\infty} s(f) d f}
$$

In fact, $f_{\text {up }}$ (positive) and $f_{\text {down }}$ (negative) are calculated separately. We have corrected for the natural differences between the upshifted and downshifted frequencies [Showen, 1979] due to the different $k$ vectors for the two spectra.

The critical point in applying this technique is the symmetry of the upshifted and downshifted spectra when the Doppler shift has been removed. These spectra will be symmetric if the distribution of electrons that interact with the plasma waves is isotropic. Since we are concerned with low-energy electrons (approximately $0.3-3 \mathrm{eV}$ ) in altitude regions (approximately $90-150 \mathrm{~km}$ ) with very large electron-electron and electron-neutral collision frequencies, transport is unimportant, and the electron distribution will be isotropic. Indeed, an examination (visual or mathematical) of the spectra presented later does show them to be symmetric.

The electron velocity averaged over the $E$ region is then given by

$$
\left\langle V_{e}\right\rangle=-\frac{c}{4} \frac{f_{\text {up }}+f_{\text {down }}}{f_{c}}
$$

While experience with $F$ region spectra [Showen, 1979] suggests that the largest contribution to the first-moment calculation comes from the peak of the layer, it is not clear that this is the case in the auroral $E$ region. Indeed, the data presented in the next section indicate that the strongest signal is at a frequency significantly below the cutoff frequency and that it originates from an altitude well above the $E$ layer peak. This average electron velocity therefore is weighted for a region above the $E$ layer peak.

\section{Measurements}

In this section we present plasma line and correlative measurements. The intent is the exploration of the capabilities of the correlator and the demonstration of the richness of both the geophysical phenomena being studied and the data set available for that study. First, we show and compare measurements from the filter bank and the FAC for March 19, 1978, UT. In the process we discuss the strengths of the two techniques and show that the new correlator technique does indeed give the same intensities as the well-established filter technique. The data presented in this part are also considered elsewhere [Oran et al., 1980] in a comparison of theory and experiment. To distinguish between the results due to the measurement technique and those due to geophysical differences, we next examine a variety of data obtained with the FAC on the night of March 16, 1978, UT. Finally, for that same night we present our initial determination of electron velocities and field-aligned currents in the premidnight diffuse aurora.

\section{a. Plasma Line Measurements With the Filter Bank and Correlator}

To show and compare the plasma line data obtained by these complementary techniques, we have chosen a period in which the signal was in as many plasma line filters as possible. In order to have this the plasma line intensity spectrum had to be particularly broad. However, under these conditions the signal was also weak and required a long integration, $23 \mathrm{~min}$. During the selected period the auroral $E$ layer was unusually stable. The peak or critical frequency and altitude are shown in Figure 4 at 1 -min intervals. It should be recalled that the plasma frequency is slightly less than the plasma line frequency [Yngvesson and Perkins, 1968]. For the data shown in this paper the difference is typically $100-300 \mathrm{kHz}$. The integrated electron density profile and the densities from the plasma line filters are shown in Figure 5. The bar at the layer peak indicates the range of density variation obtained from the 1-min profiles.

The agreement between the densities found by the two techniques, despite the geophysical variations, is remarkable because very different quantities are being averaged. For the ion component the density is found at a given altitude from the average received power (and from certain correction factors). For the electron component and the filter bank a density is found at a given altitude when there is a detectable signal at that altitude in the appropriate filter. Whether that signal can be detected becomes a question of how much it varies in altitude, how strong it is, and what fraction of the integration time the signal is present. Thus to first order the ion component provides the average density at a given altitude, while the electron component and filter bank provide the average altitude for a given density.

In Figure 6 we show the signals from the filter bank obtained at 10 of the 16 plasma line frequencies recorded. At

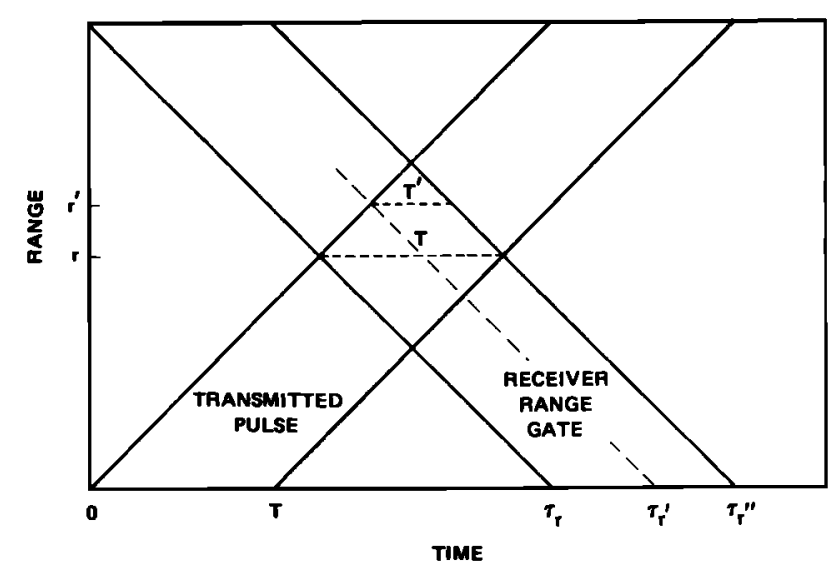

Fig. 3. Range-time diagram illustrating the measurement technique. For range $r=c \tau_{r} / 2$ associated with time $\tau_{r}$ after the beginning the transmitted pulse the signal is integrated for a time almost equal to the length of the transmitted pulse, $T=\tau_{r}{ }^{\prime \prime}-\tau_{r}$. For range $r^{\prime}=c \tau_{r}^{\prime} /$ 2 associated with a time $\tau_{r}^{\prime}$ the integration time is less, $T^{\prime}=\tau_{r}^{\prime \prime}-\tau_{r}^{\prime}$. 


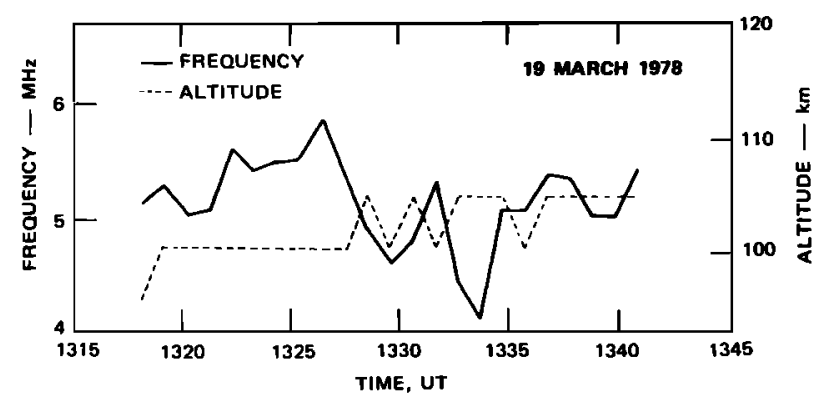

Fig. 4. Peak frequency and altitude of the auroral $E$ layer on March 19, 1978, determined with 1-min integrations. These data are from the ion component. The frequency is a continuous variable, but the altitude is quantized in steps of almost $5 \mathrm{~km}$.

-5.8 and $+5.7 \mathrm{MHz}$ the signals were not strong enough for a long enough period to detect plasma lines. In the other filters we see plasma lines of varying intensities, the strongest ones occurring at the lowest frequencies. These measurements were made with a $320-\mu$ s pulse. They show the noise level at all altitudes except for a region centered on the plasma line. Ideally, the plasma line signal would define a rectangular region the length of the radar pulse. What we see is not rectangular for three reasons. First, there is a time constant in the circuit which rounds the two edges. Second, there is a density gradient or plasma frequency gradient across the plasma line filter; the signal does not increase instantaneously from zero to its maximum value, but rather, the rise occurs over several kilometers. In this case that distance is small enough, about $1 \mathrm{~km}$, that it does not appear in these curves. Third, the layer changed during the integration time. Not only did the maximum density vary, but also the altitude at which a given smaller density occurred moved up and down. This altitude movement gives rise to more rounded edges.

We determine the magnitude of the signal by averaging the seven strongest points, subtracting the noise level, and normalizing the results to the calibration pulse. In addition, we

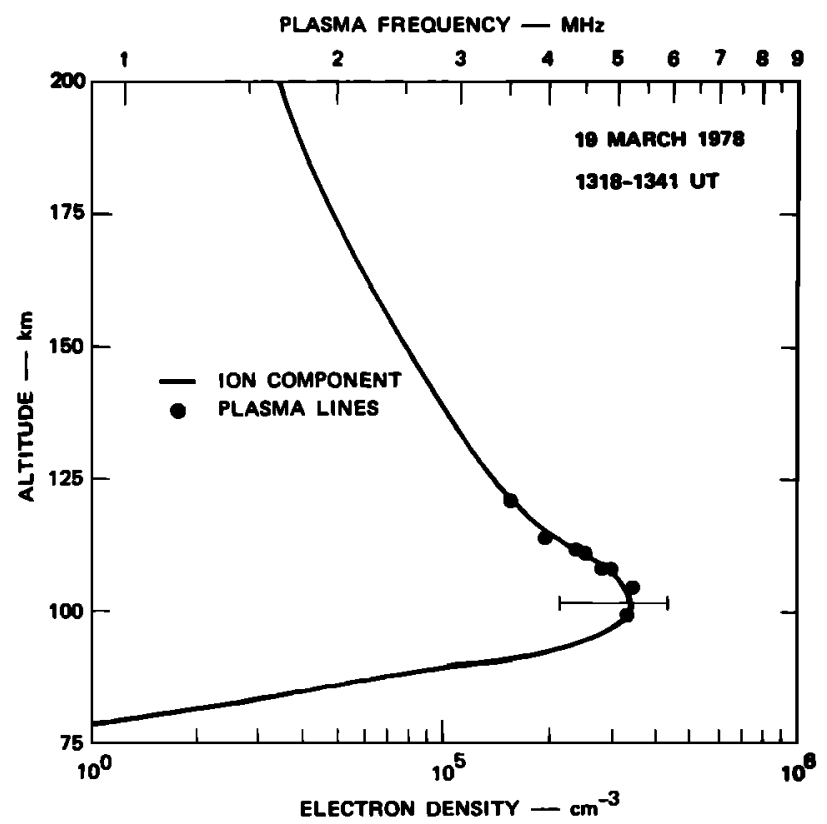

Fig. 5. Electron densities from the ion component and plasma lines on March 19,1978. The bar at the peak of the layer shows the range of variation of the peak density determined from the 1 -min integrations.

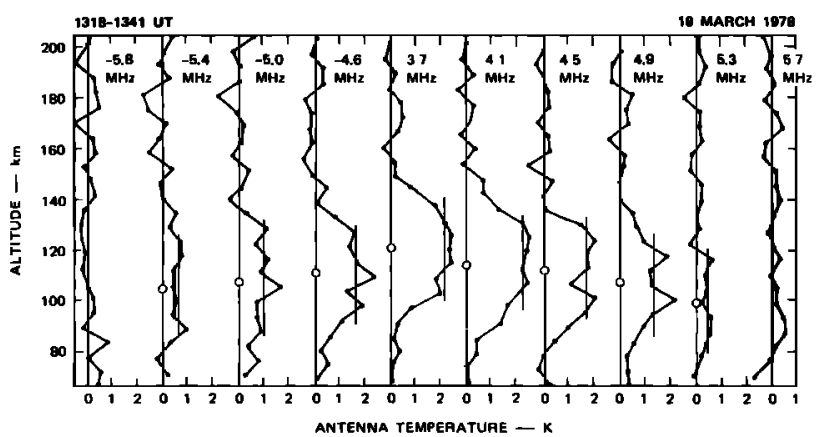

Fig. 6. Data from the plasma line filter bank on March 19, 1978. Sixteen channels of data were obtained by observing alternately every $10 \mathrm{~s}$ above and below the transmitter frequency with a set of eight filters. The signals are shown for the 10 plasma line channels with the smallest frequency offsets. Plasma lines appear in the eight of these with the smallest offsets. Their altitudes are indicated by open circles, and their intensities by vertical straight lines.

had previously made a small correction below $110 \mathrm{~km}$ for recovery; that is, below $110 \mathrm{~km}$ the noise level was slightly elevated because of increased noise in the receiver after the transmitted pulse. The recovery curve was determined by averaging several hours of plasma line data from filters whose frequencies were well above the critical frequency. We determine the uncertainty from the standard deviation of these seven points. Since the noise level is found from many more data points, it is determined to a much greater precision, and its uncertainty contributes little to the overall uncertainty in the signal strength.

The altitude of each plasma line was taken to be the midpoint between the altitudes where the signal was half of the mean value for the plasma line. A better procedure, when this operation is computerized, is the modeling of the shape of the plasma line signal and then determination of the altitude that yields the best cross correlation between the measured and modeled shapes.

An important result is apparent in these filter bank measurements. The signal appears to come only from the topside of the auroral $E$ layer. Apparently, the signal is so small from the bottomside that its magnitude is negligible when it is compared with the signal from the topside. This finding is consistent with previous measurements [Wickwar, 1978] and with the theoretical modeling of these data [Oran et al., 1980].

The result is important for the interpretation of the correlator measurements. The advantage of the correlator over the filter bank is that it can measure the spectrum continuously over a much wider frequency range with a narrower equivalent filter width; however, to gain these advantages, it sacrifices altitude resolution. The correlator, with its gate equal to the $320-\mu$ s pulse length, can, in principle, receive signals from a $96-\mathrm{km}$ range, although the altitude weighting function reduces the effective range to about $50 \mathrm{~km}$. The absence of signal from the bottomside $E$ region then assures us that even though the correlator gate encompasses all the $E$ region, most, if not all, of the signal is from the topside $E$ region. Another consequence of the long gate is that if the layer were to move in altitude during the integration period, the FAC would be more likely to obtain a detectable plasma line signal than the filter bank. This is because the signal would remain within that range gate, whereas for a filter the signal would appear in a varying set of range gates.

In Figure 7 we show examples of the 256-point autocorrela- 


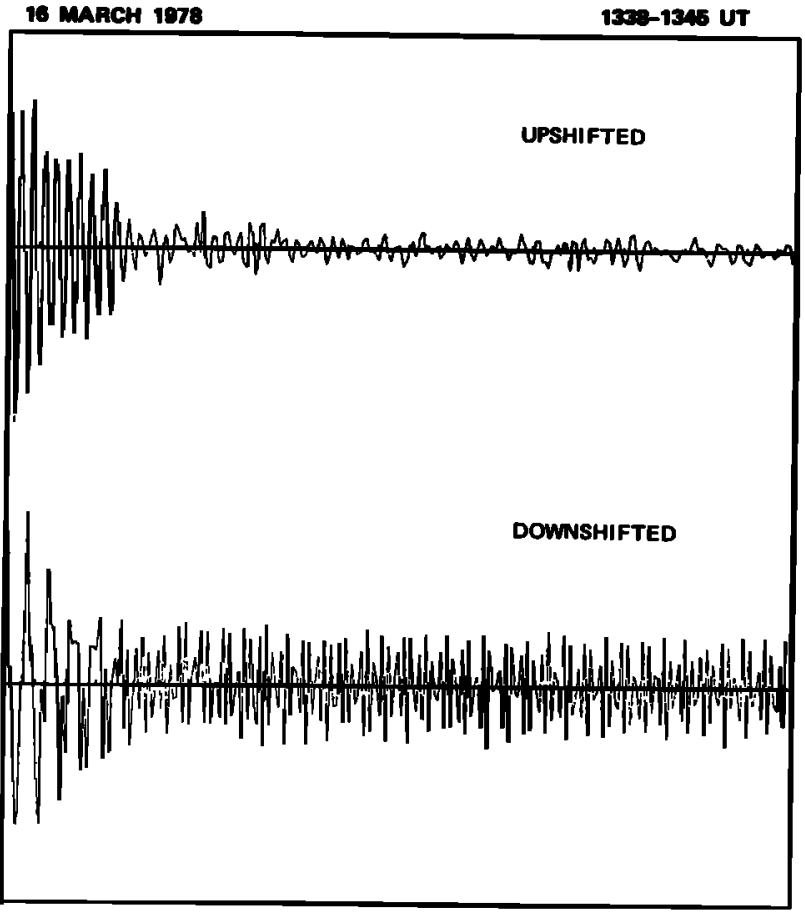

Fig. 7. Plasma line autocorrelation functions obtained with the French correlator on March 16, 1978. Each of these 256-point autocorrelation functions (ACF's) results from the subtraction of the noise ACF from the signal-plus-noise ACF. The top ACF is for the upshifted frequencies; the bottom ACF is for the downshifted frequencies but also includes the ion component. The measurements alternated every $10 \mathrm{~s}$. The data were sampled at a $15-\mathrm{MHz}$ rate with a 320 $\mu$ s or $48-\mathrm{km}$ range gate centered at $110 \mathrm{~km}$.

tion functions measured with the FAC for the upshifted and downshifted signals. While the bandwidths are the same, they are not located symmetrically about the transmitter frequency. The much stronger oscillation in the downshifted signal arises because of the presence of the ion component in these measurements. These data from March 16, 1978, were selected because they are more dramatic than the weaker signals obtained during the long average on March 19. The processing consists of subtracting the noise autocorrelation function from the signal-plus-noise autocorrelation function and then normalizing the signal spectrum to that of the calibration noise source. Before transforming from the time to the frequency domain we apply a Papoulis [1973] window to the data.

In Figure 8 we show the upshifted and downshifted spectra for the long integration period of interest along with the one standard deviation theoretical uncertainty. The spectra are weak and extend from approximately 3 to $6 \mathrm{MHz}$. A comparison of Figures 6 and 8 shows that the plasma line signal was detectable to higher frequencies with the correlator than with the filter bank. The antenna calibration has not been applied to these spectra, and the result is that the downshifted spectrum appears stronger. Within each spectrum the observed shape is determined by the shape of the plasma line intensity spectrum combined with instrumental and geophysical factors. At the low-frequency end (small offset frequencies) the altitude weighting function reduces the intensity because the corresponding altitude is well above the center of the receiver gate. At the high-frequency end, which is near the center of the receiver gate, the intensity diminishes slowly, at least

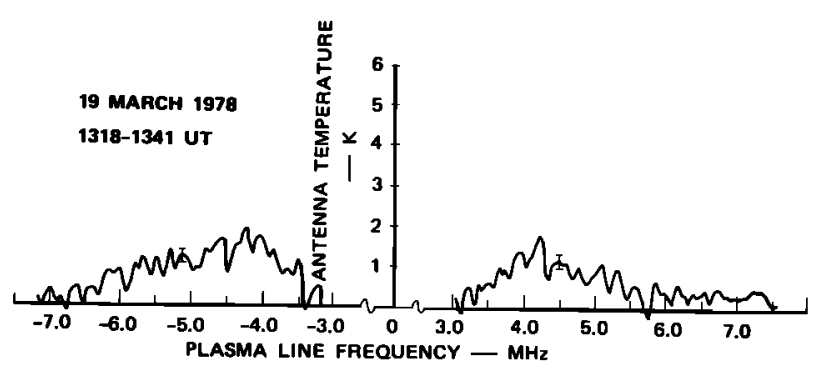

Fig. 8. Plasma line spectra obtained with the French correlator on March 19, 1978. These are the spectra of the upshifted and downshifted plasma lines in a $48-\mathrm{km}$ range gate centered at $90 \mathrm{~km}$. They have not yet been corrected for the altitude weighting function and the relative antenna calibration.

in part, because of the fraction of time that the corresponding density existed during the integration period.

In Figure 9 we show a comparison of the corrected plasma line antenna temperatures where we selected the FAC data at the frequencies of the plasma line filters and averaged them over the same bandwidth. The correlator data have now been corrected for the altitude weighting function where the altitudes were obtained from the filter bank data. These data have also been corrected for a small recovery signal that was again found from long integrations when the density was too low to give rise to a plasma line signal. Both sets of data have been corrected for the antenna calibration. The uncertainties for the filter measurements were determined from the variation of the points involved in making the estimate of the antenna temperature. The uncertainties for the correlator came from the theoretical estimate shown in Figure 8 with the altitude weighting function applied.

In this comparison we find good agreement between the intensities obtained with the correlator and the filter bank. Fur-

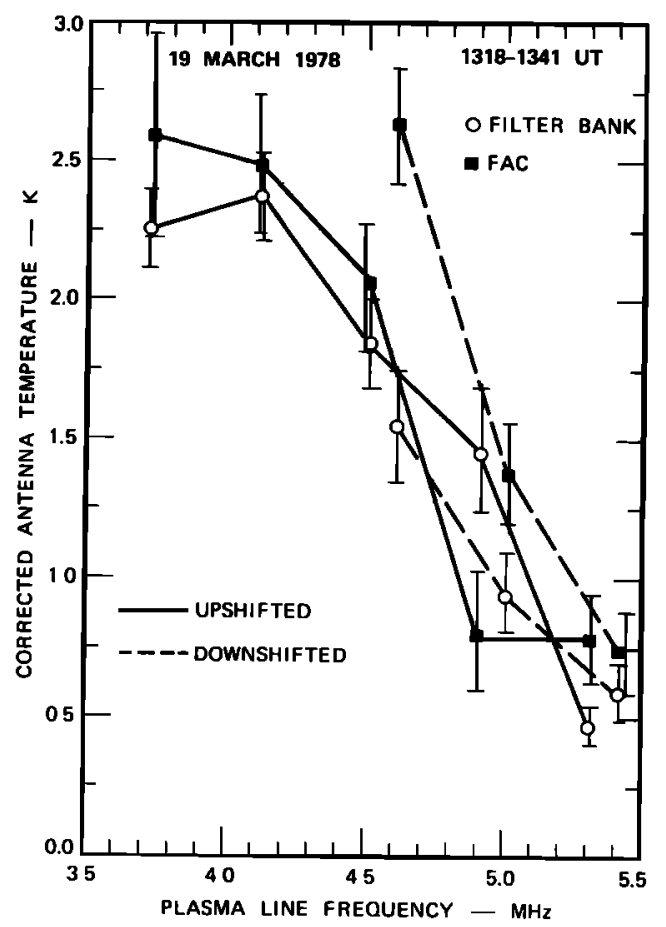

Fig. 9. Comparison of plasma line intensities obtained with the filter bank and correlator on March 19, 1978. The correlator data have been corrected for the altitude weighting function, and both sets of data have been corrected for recovery and the relative antenna calibration. 
thermore, the agreement of the low-frequency points, where the altitude weighting function requires a large correction factor, provides good verification of the theory that we presented in the last section and demonstrates that the correlator can be used for plasma line intensity measurements.

\section{b. Spectral Measurements From March 16,1978}

In this section we examine a wide range of variations in the plasma line spectra from the auroral $E$ region. We relate these to auroral conditions, in particular, the altitude and time variations of the $E$ layer. We then obtain spectra of plasma wave temperature $k T_{p}$ from the observed intensities. The $k T_{p}$ values are the physical quantities that we intend to study extensively in later work.

The varying auroral situation for the night of March 16, 1978 , can be described in a variety of ways. The magnetometer $H$ component and the riometer records for Poker Flat (4 $\mathrm{km}$ from the radar) are shown in Figures $10 a$ and $10 b$. The magnetometer is initially positive on the quiet-day curve but is negative at about 1010 and remains so with large negative bays peaking near 1210 and 1445 UT and extensive negative bay activity after 1545 UT. The riometer shows increased 30MHz absorption during the negative bay near $1210 \mathrm{UT}$ and increased absorption after $1515 \mathrm{UT}$ with a maximum near 1615 UT.

The peak density and altitude of the auroral $E$ layer is shown in Figure 10c at 1-min intervals. Superimposed on

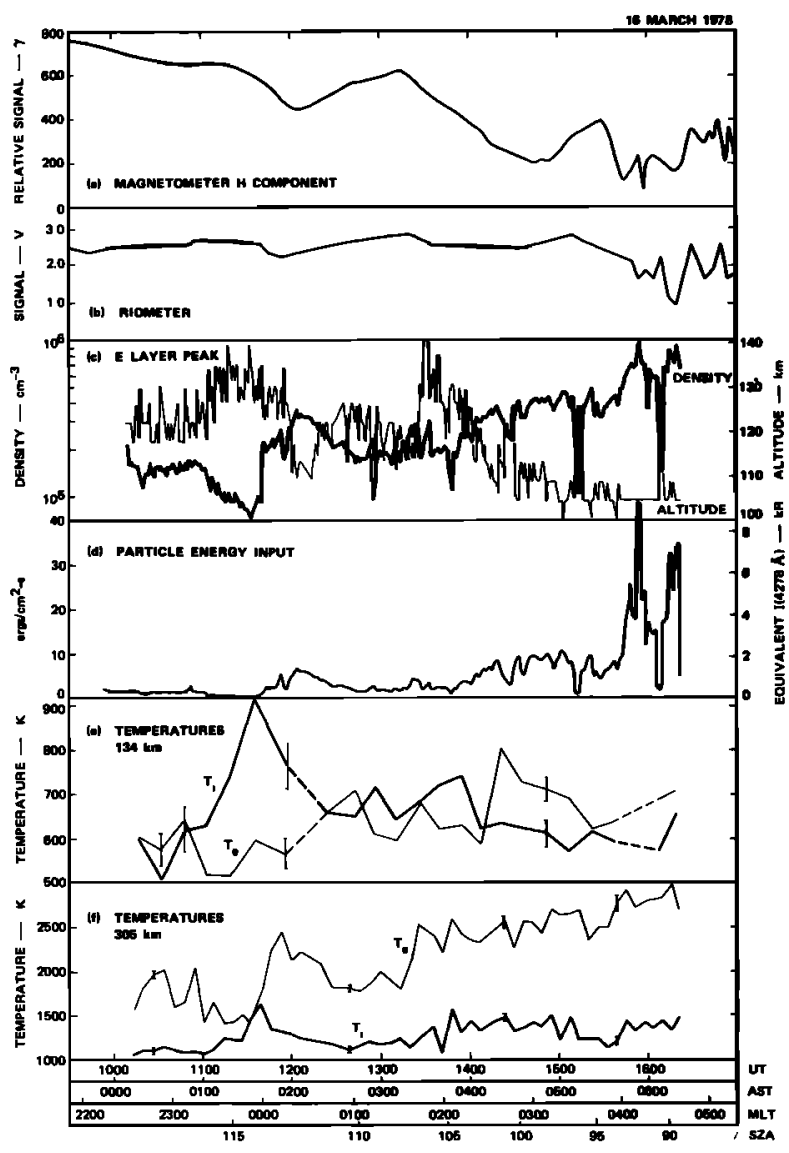

Fig. 10. Several characterizations of the auroral conditions during the plasma line observation period on March 16, 1978. Figures 10a and $10 \mathrm{~b}$ are from sensors at Poker Flat, $4 \mathrm{~km}$ from the radar. Figures $10 c-10 f$ are determined from the radar data. This figure is described in detail in the text. large fluctuations, the peak altitude decreases during the period of observation. This decrease corresponds to the usual observation of a hardening of the energetic electron flux as it passes from evening to morning. Variations in the peak density give an indication of the variation in the energetic flux. Between 1000 and 1100 UT the density has a magnitude and steadiness characteristic of the diffuse aurora. At 1430, 1515, and 1610 there are short-period reductions in electron density that are accompanied by large increases in the height of the $E$ layer peak. These distinctive features arise when the auroral precipitation becomes appreciably softer and, possibly, reduced.

From the electron density profile in the $E$ layer it is further possible to deduce the total energy brought into the atmosphere by the energetic electrons [Wickwar et al., 1975]. This energy is shown in Figure 10d. In the diffuse aurora the incident energy flux is low, about 2 ergs $\mathrm{cm}^{-2} \mathrm{~s}^{-1}$. In the vicinity of the peaks in the negative bays near 1210 and 1445 UT the energy input becomes much greater for brief intervals during the very disturbed period after 1530 ; there are peaks near 35 and 45 ergs $\mathrm{cm}^{-2} \mathrm{~s}^{-1}$. These energy inputs are very significant. In addition, the second maximum at 1615 corresponds to the biggest absorption event, indicating considerable $D$ region ionization.

Alternatively, the particle energy input and the aurora may be characterized in terms of the optical emission [Wickwar et al., 1975]. The equivalent intensity in the $\mathrm{N}_{2}{ }^{+}$band at $4278 \AA$ is also indicated in Figure $10 d$. For the band at $3914 \AA$ the intensity is $\mathbf{3}$ times as great. From these intensities it follows that the diffuse aurora is at the IBC I level, the relative maxima during the negative bays are at just below the IBC II level, and the two peaks are significantly above the IBC II level.

The electron and ion temperatures, $T_{e}$ and $T_{i}$, respectively, provide more information about the aurora. They are shown for the $E$ region, $134 \mathrm{~km}$, and the $F$ region, $305 \mathrm{~km}$, in Figures $10 e$ and $10 f$. From $T_{i}$ at $305 \mathrm{~km}$ during the diffuse aurora prior to $1100 \mathrm{UT}$ we learn that the exospheric temperature is near $1100^{\circ} \mathrm{K}$. Between 1100 and $1230 \mathrm{UT}$ the increases in both $T$, curves show a period of joule heating with a very significant maximum centered at 1140 , which coincides with a sharp increase in the particle precipitation. Just prior to this period, in agreement with the small particle precipitation, the $T_{e}$ curves show their nighttime minimum. Another joule heating event just prior to $1400 \mathrm{UT}$, when both $T_{l}$ curves have maxima, is probable. After 1330 UT the $T_{e}$ curve at $304 \mathrm{~km}$ reflects both particle precipitation and $F$ region sunrise.

Thus we have described the auroral activity during the night in terms of several different parameters. We can now choose different situations and examine what happens to the plasma line spectra. The periods centered on 1335 and 1532 UT are of interest, as can be seen in Figure $10 c$, because during the first period the $E$ layer was at its highest altitude and during the second it was at its lowest altitude. To provide as complete a description of the auroral situation as possible, Figures $11 a$ and $11 b$ show the spectra of the auroral electrons [Vondrak and Baron, 1976, 1977]. The flux is much harder and greater during the later time interval. Figures $11 c$ and $11 d$ show the measured electron density profiles from which the fluxes were derived. Not only does the later profile have a larger peak density located at a lower altitude, but it also has altitude gradients of greater magnitude on both topside and bottomside.

The upshifted plasma line intensity spectra are shown in 

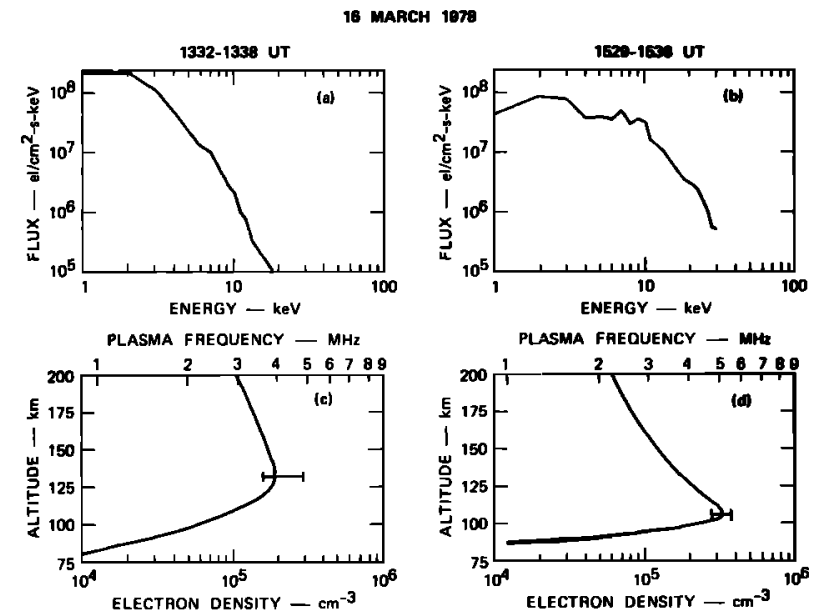

APPHOXIMATE PLASMA FREOUENCY - MHz
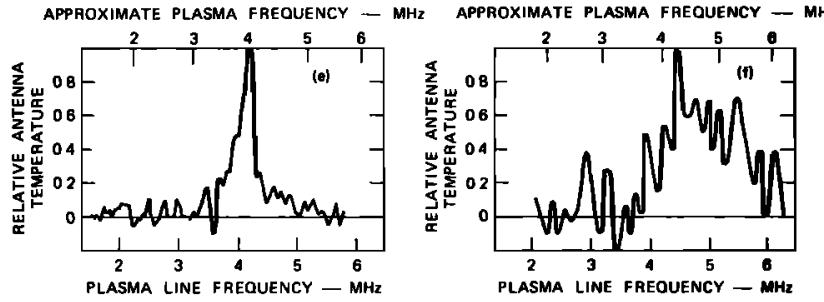

Fig. 11. Auroral conditions and plasma line data for two time periods on March 16, 1978. Figures $11 a$ and $11 b$ show the energetic electron fluxes derived from the electron density profiles in Figures $11 \mathrm{c}$ and $11 d$. The bars at the layer peaks show the range of variation found in 1-min integrations. Figures $11 e$ and $11 f$ show uplifted plasma line spectra obtained with the FAC, with the range gates set at $110 \mathrm{~km}$ and $105 \mathrm{~km}$, respectively, and a $15-\mathrm{MHz}$ sampling rate. The approximate plasma frequency was calculated assuming an electron temperature of $400^{\circ} \mathrm{K}$.

Figures 11 e and $11 f$. The spectrum is much narrower in the earlier case, $0.3 \mathrm{MHz}$ versus $1.5 \mathrm{MHz}$ at the half-power points. Since the integration times are the same, the noise fluctuations are identical. By comparing signal-to-noise ratios we deduce that the intensity is much greater in the earlier case. These differences in the observed spectra are typical and are related to the electron density distribution and the altitude of the $E$ layer. Suppose that the plasma line signal came from a limited altitude region. Then, as the magnitude of the altitude gradient became greater, the possible range of frequencies within that altitude region would also become greater. Concomitantly, there would be fewer electrons to scatter the signal in each frequency interval. Hence the signal-to-noise ratio and intensity would be reduced. In addition to these effects that the altitude gradient has upon the signal the underlying plasma wave temperature has been shown to increase with altitude up to about $120 \mathrm{~km}$ [Wickwar, 1978].

An indication of the effect that the geophysical variations have upon the observed spectra during the integration period is obtained by comparing the range of variation of the peak electron densities in Figures $11 c$ and $11 d$ to the spectra in Figures $11 e$ and $11 f$. For the earlier time period, when there is a greater density variation, there is an easily identifiable low-intensity tail at plasma frequencies above about $4.2 \mathrm{MHz}$. For the later time period there is also a low-intensity tail above a plasma frequency of about $5.2 \mathrm{MHz}$, but it is not as readily distinguishable from the rest of the spectrum.

The temporal behavior of the plasma line spectra can be examined by comparison of two periods, one with stable auroral conditions and one with dramatically changing conditions. In

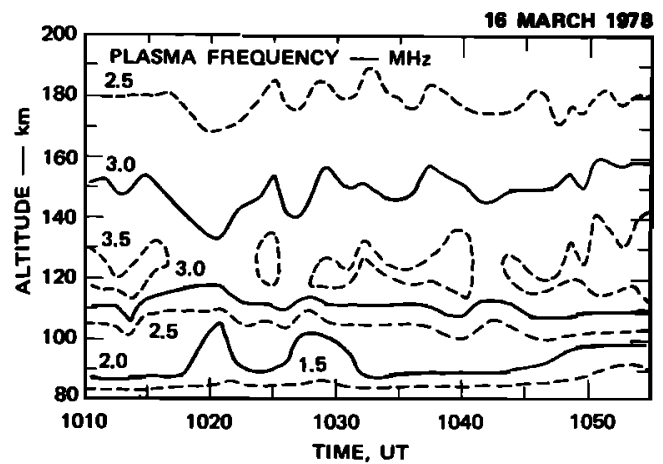

Fig. 12. Contours of $E$ region plasma frequencies during a period of diffuse aurora on March 16, 1978.

Figure 12 we show contour plots of plasma frequency obtained from a series of 1 -min integrations between 1010 and $1100 \mathrm{UT}$. This is the period of diffuse aurora previously identified in Figure 10. In Figure 13 we show the plasma line intensity spectra obtained with 6-min integrations. They are narrow and vary slowly in frequency as the maximum plasma frequency changes. They appear to be symmetric about zero, although as we pointed out earlier, the downshifted portion appears somewhat larger than the upshifted portion.

In Figure 14 we show plasma frequency contours starting 5 hours later and encompassing the second period of very intense particle precipitation discussed in conjunction with Figure 10. As the measurements from the riometer have indicated, there is considerable ionization below $90 \mathrm{~km}$. This period includes a large variation in the auroral situation. In Figure 15 we show the plasma line spectra obtained with 1min integrations. Again, the spectra appear to be symmetric about zero; however, in this case there is rapid temporal variation. After 1607 the spectra disappear for $2 \mathrm{~min}$ and then reappear with a narrow maximum near $7 \mathrm{MHz}$. After 1615 the spectra become much wider and extend up to $9 \mathrm{MHz}$.

Having examined altitude and temporal effects on the measured plasma line temperatures, we will now examine the plasma wave temperature $k T_{p}$, as discussed in section $3 b$. We examine two adjacent time periods during the diffuse aurora. The electron density profiles are shown in Figure 16a. They are very similar. The upshifted plasma line spectra are shown in Figure 16c. As is expected from the shift in the density profile, the later spectrum is shifted to higher frequencies. The maximum antenna temperature is significantly greater, and the width at half the maximum is significantly less. Both spectra have a low-intensity tail extending $200-300 \mathrm{kHz}$

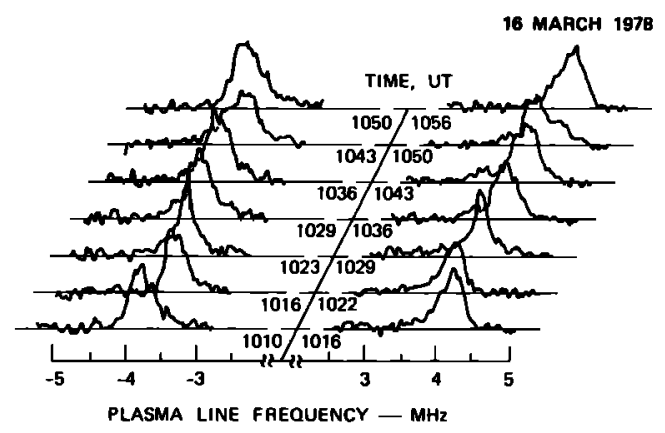

Fig. 13. Sequence of plasma line spectra during a period of diffuse aurora on March 16, 1978. The FAC range gate was centered at $120 \mathrm{~km}$, the sampling rate was $8 \mathrm{MHz}$, and the integration time was 6 min. 


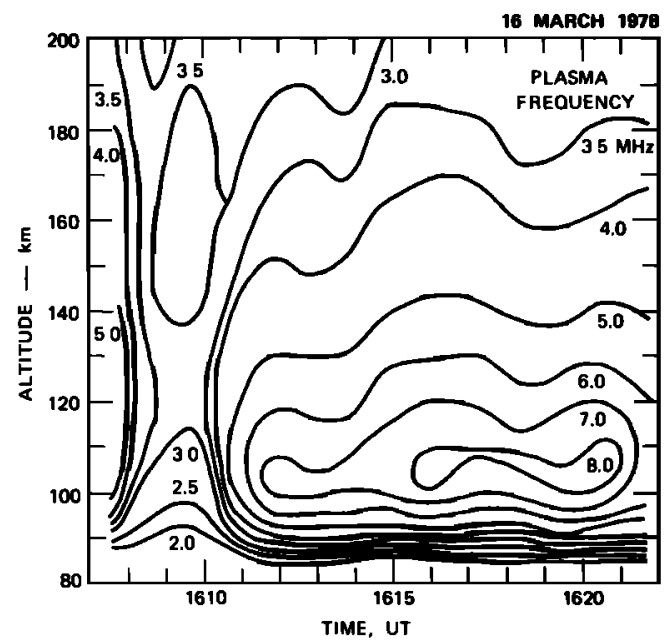

Fig. 14. Contours of $E$ region plasma frequencies during a period of active aurora on March 16, 1978.

beyond the sharp intensity decrease that occurs near $3.9 \mathrm{MHz}$. As we did previously, we attribute this tail to variation in the peak electron density during the integration period.

Much of the difference in peak intensity and width arises from the time variation of the layer. From the bars in Figure $16 a$, which show the variation in peak density, it is apparent that there was more variation during the earlier integration. This variation would lead to a smaller peak signal and wider spectrum. There is the added possibility that part of the difference may be due to the altitude dependence of the plasma line intensity because the same density in this later curve always occurs at a higher altitude.

To find $k T_{p}$ from the plasma line intensity spectrum, (7), we need to associate an altitude with each frequency. Unfortunately, this task is not as easy as converting a plasma line frequency to density and then using the density profile. Because of the accumulative effects of the averaging discussed previously, the temperature dependence of the scattering cross section, and the absolute calibration of the radar the plasma line frequency is better known than the absolute scale of the density profile. We therefore use the plasma line data to normalize the density profile. Usually, we would do this with the data from the filter bank; however, there are no such data on this occasion. Thus we use the FAC data, applying the procedure discussed below.

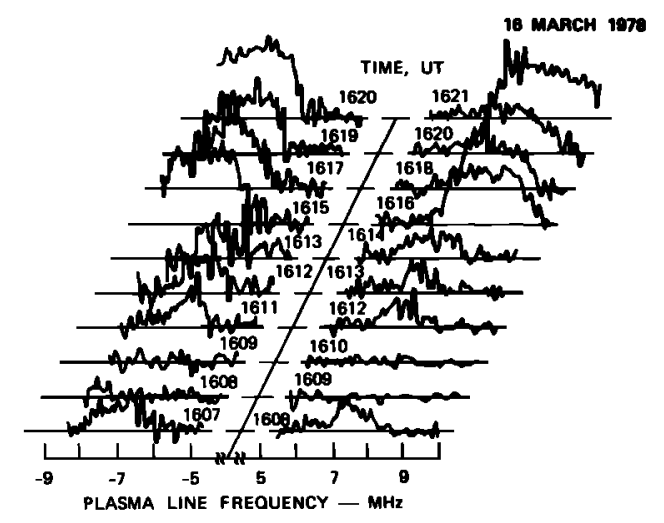

Fig. 15. Sequence of plasma line spectra at 1 -min intervals during a period of active aurora on March 16, 1978. The FAC range gate was centered at $105 \mathrm{~km}$, the sampling rate was $15 \mathrm{MHz}$, and the integration time was I-min.
In the $F$ region at Chatanika and elsewhere there is a very sharp cutoff to the plasma line spectrum at the peak frequency. It is all the more dramtic because the maximum signal is at the low-frequency side of the cutoff [cf. Showen, 1979]; hence it is easy to determine the peak density and scale the ion component density profile. In the auroral $E$ region the situation is different: the cutoff is less sharp for two reasons. First, there is temporal variation of the peak density during the integration time. We have already discussed some of the effects of this variation. Second, the maximum signal may come from a higher altitude than the peak. This appears to be the case for the data from March 19, 1978, shown in Figures 4, 6, and 9. The maximum signal is near $4.0 \mathrm{MHz}$, and the signal is already significantly smaller by $4.5 \mathrm{MHz}$. Both the frequencies are considerably less than the peak frequency. Indeed, both these frequencies are sufficiently below the peak frequency that the intensities should be little affected by the variation of the layer during the integration time. This also appears to be the case for the data from March 16, 1978. In the short 1-min integrations in Figure 15 the intensity falls off gradually rather than precipitously at the high-frequency end of the spectra.

As for the data in Figure 16c, we have adopted the following procedure: by extending the main decline to zero intensity (at the frequencies shown by the arrows with open symbols) we obtained the peak frequency and hence the peak density. We then scaled the ion component density profile and attributed an altitude to each datum point in the spectrum, enabling the plasma wave temperatures to be calculated by using (7). The $k T_{p}$ values were then calculated for the data points shown in Figure $16 c$ after correction for the altitude weighting function shown in Figure 16a. The resultant $k T_{p}$ values are presented versus altitude in Figure $16 b$ and versus plasma frequency (and phase energy) in Figure $16 d$.

Thus we have obtained $k T_{p}$ values ranging from 0.1 to 1.1 $\mathrm{eV}$ over an altitude range from 122 to $150 \mathrm{~km}$ and a phase energy range from 0.45 to $0.65 \mathrm{eV}$. The $k T_{p}$ spectra for the two periods are similar as they reach their maxima near the highfrequency or high-energy end. The shift in location of the maximum is in accord with the change in the auroral $E$ layer. The altitude profiles of $k T_{p}$ are in reasonable agreement and show $k T_{p}$ increasing from the peak of the $E$ layer to a maximum about $10 \mathrm{~km}$ higher and then falling off to higher altitudes. The differences between the curves give an indication of the uncertainty, including contributions from geophysical variations.

The important physical result here is that the peak of the $k T_{p}$ profile is at a greater altitude than the peak of the $E$ layer. As will be mentioned in section 5 , we believe that this difference in altitudes indicates that the secondary electron flux is greater at altitudes above the peak of the layer.

For the two peaks to be at the same altitude a frequency near the peak of the observed signal spectrum in Figure $16 c$ would have to be identified with the peak of the $E$ layer. This is unlikely. First, it would be difficult to account for the signal at frequencies above the peak with a spectrum having a sharp cutoff and the small observed peak frequency variation. Second, other data for which the altitudes are well determined, such as the data from March 19, 1978, that we have already discussed, are inconsistent with this interpretation; recall that the lower frequencies during a given measurement refer to greater heights because the signal was found only on the topside of the layer. We have further explored the effect of an er- 
ALTITUDE WEIGHTING FUNCTION
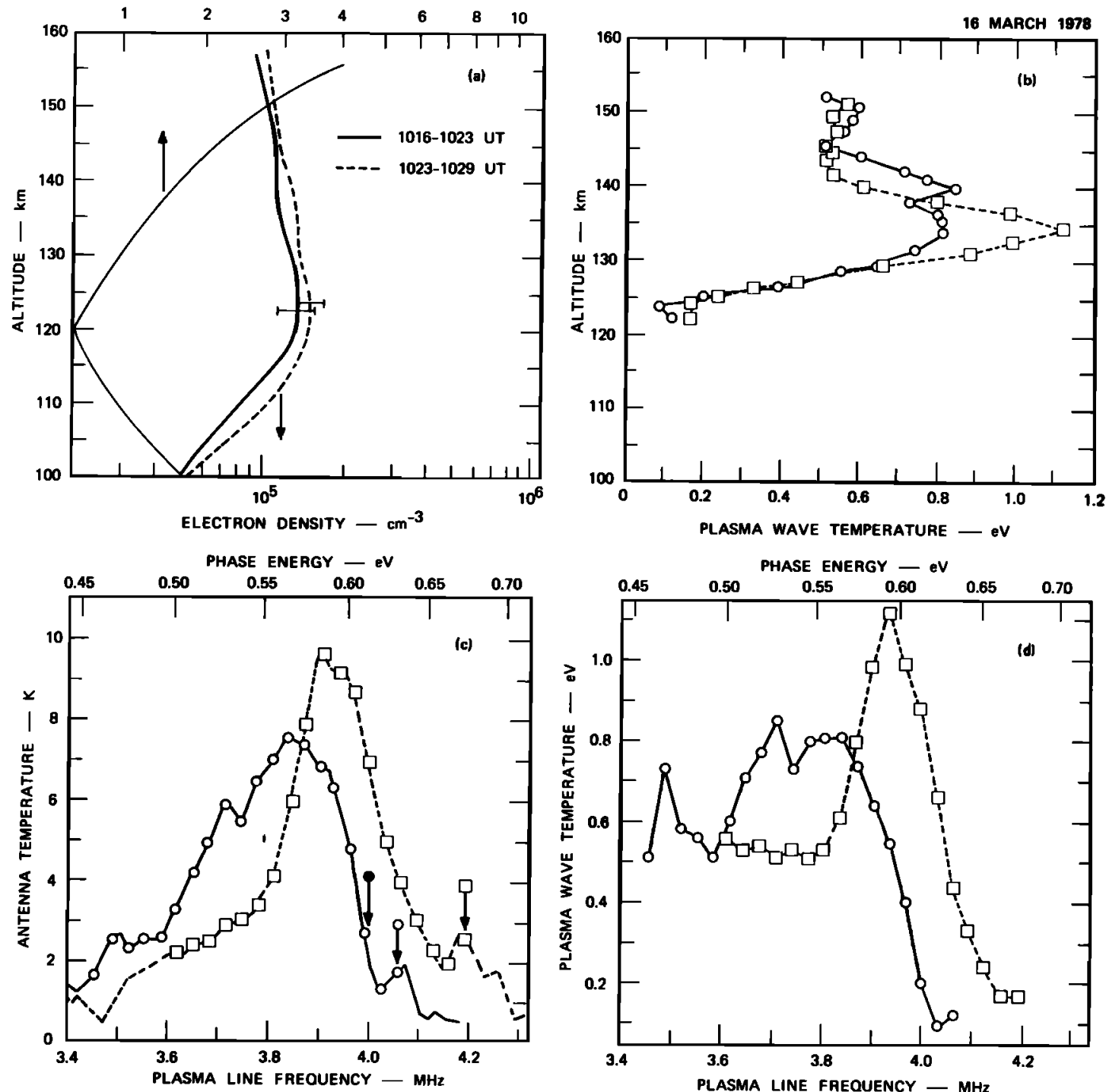

Fig. 16. Plasma line spectra and derived plasma wave temperatures during two periods of diffuse aurora on March 16, 1978. (a) Electron density profiles. The bars near the layer peaks show the ranges of variation found in 1-min integrations. The FAC range gate was centered at $120 \mathrm{~km}$, giving rise to the altitude weighting function associated with the top scale. (b) Plasma wave temperature profiles derived from the spectra in Figure 16c. (c) Two observed upshifted spectra of the plasma lines. Every other data point is shown for a portion of the spectra starting with the point, identified by an arrow and open symbol, that is associated with the layer peak in Figure 16a. (d) Plasma wave temperature spectra.

ror in the peak frequency determination by assigning the frequency marked by the arrow with the solid symbol in Figure $16 c$ to the peak frequency and deriving the $k T_{p}$ values. In frequency the resultant $k T_{p}$ spectrum is similar to that shown in Figure $16 d$, but the values are smaller. In altitude the $k T_{p}$ profile is similar to that shown in Figure $16 b$ but is shifted down in altitude by about $4 \mathrm{~km}$. Thus while the detailed values could change, the important physical result would remain unchanged.

\section{c. Electron Velocity and Current Parallel to the Magnetic Field}

In order to attempt this measurement we used a slower sampling rate to obtain greater frequency resolution. By changing from $15 \mathrm{MHz}$ to $8 \mathrm{MHz}$ the equivalent filter changed from 30 to $16 \mathrm{kHz}$. The measurements were made during a period of good signal-to-noise ratio and stable dif- fuse aurora, 1010 to 1055 UT on March 16, 1978. Data from this period have already been discussed extensively. The plasma frequencies and plasma line intensities have been presented in Figures 12 and 13.

We applied the procedure described in section $3 c$ to find the Doppler shifts. Since the spectra appear to be truly symmetric, the procedure is applicable. The results of this analysis, the combined frequency shifts, are shown in Figure 17. Each point at 2-min intervals is the result of a 6-min integration. The statistical uncertainty is of the same order as many, but not all, the measurements. If we assume that the current in a stable aurora is almost constant, instead of looking at the individual points we can average all the data. We find that the combined frequency shift $f_{\text {up }}+f_{\text {down }}$ is approximately -10.6 $\mathrm{kHz}$ with an estimated one standard deviation uncertainty of $8 \mathrm{kHz}$. Again, this result is significant. This frequency shift gives rise to an electron velocity of $0.6 \mathrm{~km} / \mathrm{s}$. The ion velocity parallel to the magnetic field is negligible in comparison. For 


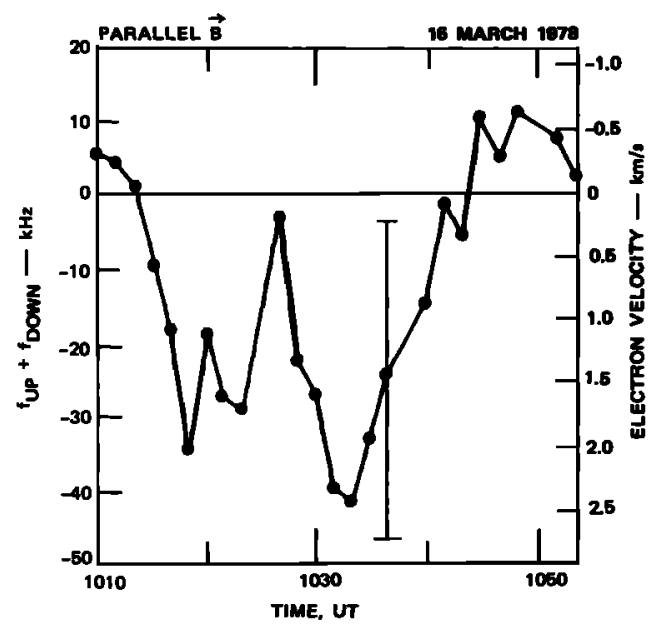

Fig. 17. Doppler shift of the plasma line spectra or electron velocity during the diffuse aurora. Although separated by $2 \mathrm{~min}$, each datum point was found using a 6-min integration. The Doppler shift was determined from the first moment.

an electron density of $10^{5} \mathrm{~cm}^{3}$ this velocity implies a current down the magnetic field line of the order of $10 \mu \mathrm{A} / \mathrm{m}^{2}$.

As we noted in section $3 c$, the current determination applies to a region above the $E$ layer peak because it is there that the observed signal originates. In Figure $16 b$, which was also discussed earlier and is from the time period of the current determination, we see that the peak of the $k T_{p}$ profile is between 130 and $135 \mathrm{~km}$. A comparison of the squares and circles in Figures $16 c$ and $16 d$ further indicates that the observed spectrum also has its peak between 130 and $135 \mathrm{~km}$, while Figure $16 a$ shows that the $E$ region density peak is at $122 \mathrm{~km}$. Therefore the averaged current is from a region about $10 \mathrm{~km}$ above the peak of the $E$ region in this example.

The magnitude of this current is reasonable in comparison to satellite measurements [Potemra, 1977], but we have no correlative measurements to verify it. However, downward fieldaligned currents are expected statistically in the evening sector in the diffuse aurora [Kamide and Rostoker, 1977] or in the evening sector near the equatorward portion of the auroral oval [Potemra, 1977]. These two descriptions are equivalent in that the diffuse aurora occurs in the equatorward portion of the oval. The low and relatively constant electron densities that we measure in the auroral $E$ layer (Figures 10 and 12) clearly indicate that we are in the region of diffuse aurora. This conclusion is further supported by the equivalent 4278- $\AA$ intensity (Figure 10d), which is typical of the diffuse aurora. That we are in the evening sector is supported by the fact that the observations were made about 1 hour before magnetic midnight. However, in terms of the eastward electrojet our location in the evening sector is not as clear because the magnetometer $H$ component is just negative of the quiet-time curve.

As we improve this technique and gain more confidence in it, we expect to define where and under what conditions Birkeland currents occur. At least we will be able to measure that part of the Birkeland current that is carried by thermal electrons. Suitable comparisons with magnetometer experiments could then resolve the question of whether ions contribute significantly to these currents.

\section{Discussion AND CONClUSION}

In the spring and fall of 1978 we made a series of plasma line observations in the auroral region at Chatanika, Alaska.
While successful measurements had previously been made [Wickwar, 1978], this series of observations was unique in that it used a high-speed correlator as well as the usual filter bank to measure the plasma line intensity spectra. In addition, more simultaneous ion component measurements that are necessary for the data interpretation were made. Altogether, a greater number of different, yet simultaneous, power and spectral measurements were made with the radar than had been undertaken before.

We have developed the theoretical basis for obtaining the intensity spectrum using correlator measurements and a monostatic radar. A comparison of the filter bank and correlator measurements proves that the correlator can indeed be used successfully to measure plasma line spectra. The spectra measured in this manner have the advantage that they cover a continuous wide frequency band with better spectral resolution than the filter bank alone. The filter bank nicely complements these spectra by providing range information for scaling the density profiles and for separating intensities from the topside and bottomside of the layer.

A large series of observations were made under a variety of conditions during both the day and the night. Different observations from the auroral $E$ layer have been presented to show the richness of the phenomenon that we observed; specifically, they enable us to examine the shape and variations in shape of the spectra. In order to relate these measurements to the auroral situation we have presented a particularly wide range of correlative measurements.

As was previously found [Wickwar, 1978], the plasma line intensity on the bottomside of the $E$ layer was found to be insignificant in comparison with the topside intensity. Theoretical calculations [Oran et al., 1980] are consistent with this finding. As is expected, the observed spectra were found to be wider and weaker for large density gradients than for small gradients. Unlike daytime spectra excited by photoelectrons, the signal strength does not usually approach a strong maximum followed by a sharp cutoff at the high-frequency end of the spectrum. Instead, there is a more modest maximum followed by a more gradual falloff. Consequently, the observed intensities and the derived plasma wave temperatures have their maxima significantly above the $E$ layer peak. Again, theoretical calculations [Oran et al., 1980] are consistent with this finding.

First, at the lower altitudes in the topside $E$ region the sharp rise in $k T_{p}$ is very similar to that found by Wickwar [1978]. Then, the rate of increase with altitude was found to be inversely proportional to the variation of neutral density with altitude. This behavior of $k T_{p}$ was attributed to the behavior of the steady state secondary electron flux, which is primarily controlled by the ratio of the production rate to the energy loss rate to neutrals. In those earlier data the situation was simplified because the ionization rate was nearly constant over the altitude range considered. In these data, where the production rate decreases with altitude, the explanation of the $k T_{p}$ altitude behavior is the same but with the added implication that the energy loss rate to neutrals must decrease with altitude over the observed altitude region. Since phase energy decreases with altitude, this is equivalent to saying that the loss rate to neutrals increases with energy in the energy region near $1 \mathrm{eV}$. Second, at the higher altitudes there is a sharp fall in $k T_{p}$ with altitude. This behavior was also seen by Wickwar [1978] and was attributed to the increased importance of the Maxwellian term in the enhancement equation at higher alti- 
tudes and hence larger electron temperatures. That effect would be even more pronounced here because the phase energy is decreasing with altitude. As more of the data are analyzed and the results modeled, we will be better able to test this interpretation and fill in more of the details.

In addition to these extensive observations of the plasma line spectra enhanced by secondary electrons in the auroral $E$ layer we have also made the first observations in the auroral region of $E$ region and $F$ region plasma lines enhanced by photoelectrons. These plasma lines will be examined in a subsequent article.

Besides examining the shape of the plasma line spectra we have determined the Doppler shift of the spectra and hence the drift velocity of the ambient electrons. By combining the electron velocity with the simultaneous measurements of ion velocity and electron density in the topside $E$ region we have made the first radar measurements of the Birkeland currents carried by ambient electrons.

We have given an overview of the joint American-French plasma line experiments conducted at Chatanika in March and November 1978. We are continuing the data analysis and expect to concentrate our efforts in two directions. The first involves the differential fluxes of low-energy electrons in the $E$ layer and the factors such as the neutral atmosphere that affect these fluxes. The second involves the Birkeland currents. We expect to improve the analysis procedure, make comparisons to other measurements such as those obtained by the Alaskan magnetometer chain and satellites, and study the conditions under which these currents occur and vary.

\section{Appendix A: Theory of Plasma line Correlation MEASUREMENTS}

The scattered signal at point $\mathrm{r}$ and time $t$, applying the first Born approximation to the ionospheric plasma, is

$$
\begin{aligned}
& E_{s}(\mathbf{r} ; t)=-r_{e} \frac{\exp (-i \mathbf{k r})}{r} \exp \left(i \omega_{c} t\right) \sin \chi \\
& \cdot \int P\left[t+\tau_{r}-\left(\hat{a}_{k}-\hat{a}_{r}\right) \frac{\mathbf{r}^{\prime}}{c}\right] \times \Delta N_{e}\left(\mathbf{r}^{\prime} ; t\right) \exp \left(-i \mathbf{k}_{\mathrm{eff}} \mathbf{r}^{\prime}\right) d \mathbf{r}^{\prime}
\end{aligned}
$$

where

$r_{e}$ electron radius;

$f_{c}$ transmitter frequency;

$\omega_{c}=2 \pi f_{c}$;

k transmitted wave vector;

$\mathbf{k}_{r}=$ received wave vector;

$\mathbf{k}_{\text {eff }}=\mathbf{k}-\mathbf{k}_{r}$;

$\hat{a}_{k} \quad$ unit vector along $\mathbf{k}$;

$\hat{a}_{r}$ unit vector along $\mathbf{r}$;

$\tau_{r}$ time from transmission to reception;

$P$ envelope of the transmitted signal;

$\Delta N_{e}$ electron number density fluctuation;

$\chi$ electron scattering angle.

To obtain a one-dimensional equation, we choose the $z$ axis along $k_{e r r}$ and simplify the equation by integrating $\Delta N_{e}$ over a surface $S$ in the $x y$ plane

$$
\overline{\Delta N_{e}}(z ; t)=\frac{1}{A_{r}} \iint \Delta N_{e}(z ; \mathbf{S} ; t) d \mathbf{S}
$$

where $A$, is the cross section of the scattering volume. We obtain

$$
\begin{array}{r}
E_{s}(\mathbf{r} ; t)=-r_{e} \sin \chi \exp \left(i \omega_{c} t\right)\left(\frac{A_{r}}{r}\right) \int P\left(t+\tau_{r}-2 \frac{z}{c} \sin \frac{\theta}{2}\right) \\
\times \overline{\Delta N_{e}}(z ; t) \exp \left(-i k_{\mathrm{eff}} z\right) d z
\end{array}
$$

after dropping the phase factor and where $\theta$ is the angle between $\hat{a}_{k}$ and $\hat{a}_{r}$.

The correlation function of the received signal is

$$
\boldsymbol{R}_{s}(\tau ; t)=\mathbf{E}\left\{E_{s}(\mathbf{r} ; t) E_{s}^{*}(\mathbf{r} ; t-\tau)\right\}
$$

The correlation function of density fluctuations in a statistically stationary medium is

$$
R_{\overline{\Delta N_{e}}}(\tau ; \bar{z}, \Delta z)=\mathbf{E}\left\{\overline{\Delta N_{e}}(\bar{z}+\Delta z ; t+\tau) \overline{\Delta N_{e}}(\bar{z} ; t)\right\}
$$

We assume that the medium has uncorrelated scatterers because it decorrelates rapidly in $\Delta z$, typically of the order of a Debye length. Hence we obtain

$$
\begin{aligned}
& R_{s}(\tau ; t)=r_{e}^{2} \sin ^{2} \chi \frac{A_{r}^{2}}{r^{2}} \int P\left(t+\tau_{r}-2 \frac{\bar{z}}{c} \sin \frac{\theta}{2}\right) \\
& \times P^{*}\left(t+\tau_{r}-\tau-2 \frac{\bar{z}}{c} \sin \frac{\theta}{2}\right) R_{\overline{\Delta N_{e}}}\left(\tau ; \bar{z} ; k_{\mathrm{eft}}\right) d \bar{z}
\end{aligned}
$$

where

$\boldsymbol{R}_{\overline{\Delta N}}\left(\tau ; \bar{z} ; k_{\mathrm{eff}}\right)=\int\left[\exp \left(-i k_{\mathrm{eff}} \Delta z\right)\right] \boldsymbol{R}_{\overline{\Delta N}}(\tau ; \bar{z} ; \Delta z) d \Delta z$

This equation, which is the same as that obtained by Farley [1969] and Rino [1972], is the starting point of our analysis.

We now calculate the correlation function for our correlator for a rectangular pulse of duration $T$ and a range gate also of duration $T$. First, we change variables and drop the initial constant. This gives

$$
\begin{aligned}
R_{s}(\tau ; t) \propto \int P\left(t-t_{d}\right) P^{*}(t- & \left.\tau-t_{d}\right) R_{\Delta N_{f}}\left(\tau ; \frac{c^{\prime} t_{d}}{2}\right) d t_{d} \\
& =\int_{\tau}^{T} R_{\Delta N_{e}}\left[\tau ; \frac{c^{\prime}(t-p)}{2}\right] d p
\end{aligned}
$$

where

$$
\begin{aligned}
& c^{\prime}=\frac{c}{\sin (\theta / 2)} \\
& 0<\tau<\tau_{\max }
\end{aligned}
$$

( $\tau_{\max }$ is the maximum delay in the correlation function and between the onset of sampling and calculation), and

$$
t=\tau_{r}+t^{\prime}+\tau_{\max } \quad t^{\prime} \geq 0
$$

Usually, for the correlation function we have $\tau_{\max } / T \ll 1$. Indeed, it is usually between 0.1 and 0.05 . Thus for our plasma line measurements the difference between the integrals $\int_{0}^{T}$ and $\int_{\tau_{\max }}{ }^{T}$ becomes negligible. To a good approximation, (A8) becomes

$$
R_{s}(\tau ; t) \propto \int_{\tau_{\max }}^{T} R_{\overline{\Delta N_{e}}}\left[\tau ; \frac{c^{\prime}(t-p)}{2}\right] d p
$$


Since the correlator works in a discrete manner and makes a temporal average, our estimate of the aurocorrelation function becomes

$\hat{R}_{s}\left(n \Delta t ; \tau_{r}+k \Delta t+M \Delta t\right) \simeq \frac{1}{N} \sum_{k=0}^{N-1} E_{s}\left(r ; \tau_{r}+k \Delta t+M \Delta t\right)$

$$
\cdot E_{s}^{*}\left(r ; \tau_{s}+k \Delta t+M \Delta t-n \Delta t\right)
$$

where

$$
\begin{array}{cc}
n \Delta t=\tau & k \Delta t=t^{\prime} \\
M \Delta t=\tau_{\max } & N=(T-M \Delta t) / \Delta t
\end{array}
$$

$N$ is the number of measurements of each lag product. In this case we have

$$
\begin{gathered}
\mathbf{E}\left\{\hat{R}_{s}\right\}=\frac{1}{N} \sum_{k=0}^{N-1} \int_{\tau_{\max }}^{T} R_{\overline{\Delta N_{e}}}\left[n \Delta t ; \frac{c^{\prime}\left(k \Delta t+M \Delta t+\tau_{r}-p\right)}{2}\right] d p \\
\simeq \frac{1}{N} \sum_{m=-(N-1)}^{N-1}\left(1-\frac{|m|}{N}\right) R_{\overline{\Delta N_{e}}}\left[n \Delta t ; \frac{c^{\prime}\left(\tau_{r}+m \Delta t\right)}{2}\right]
\end{gathered}
$$

This equation relating the medium and measured autocorrelation functions is our basic result. An intuitive discussion of it is given in section $3 a$ in conjunction with Figure 3.

The Fourier transform of (A11) relates the expected value of the measured spectrum to the spectrum of the medium. Equation (3) gives the result for the whole altitude region sampled, and (4) gives the result for a small altitude region.

We also have to consider the effects of the receiver bandwidth upon the received plasma line signal. The receiver system, up to the point where the correlation function is formed, can be represented by a single filter with the impulse response

$$
h(t) \stackrel{F T(t)}{\rightleftarrows} H(f)
$$

The resultant correlation function is analogous to that given by (A8):

$R_{s}(\tau ; t) \propto \iint \theta\left(t-t_{d} ; f\right) \theta^{*}\left(t-t_{d}-\tau ; f\right) \varphi\left(f ; \frac{c^{\prime} t_{d}}{2}\right) d t_{d} d f$

where

$$
\begin{gathered}
\varphi(f ; z) \stackrel{F T(\tau)}{\rightleftarrows} R_{\overline{\Delta N_{e}}}(\tau ; z) \\
\theta(t ; f)=\int_{-\infty}^{\infty} H\left(f^{\prime}\right) p\left(f^{\prime}-f\right) \exp \left(2 \pi i f^{\prime} t\right) d f^{\prime} \\
P(t) \stackrel{F T(t)}{\rightleftarrows} p(f)
\end{gathered}
$$

and $P(t)$ is the envelop of the transmitted signal. For plasma lines, unlike the ion component, the bandwidth of $p(f)$ is very much smaller than that of $H(f)$.

Applying the impulse function, we obtain

$$
\begin{aligned}
\theta(t ; f) \cong H(f) P(t) e^{2 \pi i f t} \\
R_{\bar{s}}(\tau, t)=\iint|H(f)|^{2} P_{T}\left(t-t_{d}\right) P_{T}^{*}\left(t-t_{d}-\tau\right) \\
\cdot e^{2 \pi f f \tau} \varphi\left(f ; \frac{c^{\prime} t_{d}}{2}\right) d f d t_{d} \\
=\iiint|H(f)|^{2} P_{T}\left(t-t_{d}\right) P_{T}^{*}\left(t-t_{d}-\tau\right)
\end{aligned}
$$

$$
\begin{aligned}
& \cdot e^{2 \pi f f^{\prime}} R_{\Delta N_{f}}\left(\tau^{\prime} ; \frac{c^{\prime} t_{d}}{2}\right) e^{-2 \pi f f^{\prime}} d \tau^{\prime} d f d t_{d} \\
= & \iint R_{H}\left(\tau-\tau^{\prime}\right) P_{T}\left(t-t_{d}\right) P_{T}^{*}\left(t-t_{d}-\tau\right) R_{\overline{\Delta N_{c}}} \\
& \cdot\left(\tau^{\prime} ; \frac{c^{\prime} t_{d}}{2}\right) d \tau^{\prime} d t_{d}
\end{aligned}
$$

where

$$
R_{H}(\tau)=\int h(t+\tau) h^{*}(t) d t
$$

Since $R_{H}(\tau)$ is much narrower than $P(t)$, we can write

$$
R_{H}\left(\tau-\tau^{\prime}\right) P_{T}\left(t-t_{d}-\tau\right) \simeq R_{H}\left(\tau-\tau^{\prime}\right) P_{T}\left(t-t_{d}-\tau^{\prime}\right)
$$

then

$$
\begin{aligned}
& R_{\dot{s}}(\tau, t) \simeq \iint R_{H}\left(\tau-\tau^{\prime}\right) P_{T}\left(t-t_{d}\right) P_{T}^{*}\left(t-t_{d}-\tau^{\prime}\right) \\
& \cdot R_{\overline{\Delta N}}\left(\tau^{\prime} ; \frac{c^{\prime} t_{d}}{2}\right) d \tau^{\prime} d t_{d}=\int R_{H}\left(\tau-\tau^{\prime}\right) R_{s}\left(\tau^{\prime} ; t\right) d \tau^{\prime}
\end{aligned}
$$

This last equation is the convolution of the correlation function of $R_{H}(\tau)$ and the correlation function of the medium smeared over altitude, (Al1).

By performing a Fourier transform on (A15) we obtain the spectrum

$$
S_{s}(f ; t) \simeq|H(f)|^{2} S_{s}(f ; t)
$$

The influence of the filter is the same as that for the case of stationary signals. Thus the measured spectrum is the product of the filter spectrum and the composite spectrum of the medium, (3).

Acknowledgments. We would like to thank the staff members at Grenoble and SRI International whose considerable assistance made these observations possible. In particular, we would like to thank Bernard Chabert, Murray Baron, Howard Burch, Bruce Craig, Ragni Pasturel, and Juris Petriceks for engineering and system design; Charles Dawson for the observation program; John Kelly for the antenna calibration and much else; Mary McCready, Chuck Code, and Carl Dean Feken for their assistance at Chatanika; Lorie McNiel and Carol Leger for programing; and Nancy Wallace Hiles for data reduction. The French portion of the experiment was supported by the Actions Thématiques Programmées of the Centre National de la Recherche Scientifique for the International Magnetospheric Study. It was supported as well by the Centre de Recherches en Physique de l'Environnement Terrestre et Planetaire and by the Centre National d'Etudes des Télécommunications. The American portion was supported by grant ATM78-00129 from the Aeronomy Program, Division of Atmospheric Sciences, National Science Foundation. In addition, the Chatanika radar is operated by SRI International under contract DNA001-77-C-0042 from the Defense Nuclear Agency and grant ATM72-01644-A05 from the Divison of Atmospheric Sciences, National Science Foundation.

The Editor thanks J. V. Evans and D. T. Farley for their assistance in evaluating this paper.

\section{REFERENCES}

Baron, M. J., Electron densities within aurorae and other auroral $E$ region characteristics, Radio Sci., 9, 341-348, 1974.

Baron, M. J., The Chatanika radar system, in Radar Probing of the Auroral Plasma, Proceedings of the EISCAT Summer School, Tromsd, Norway, June S-13, 1975, edited by A. Brekke, pp. 103141, Scandinavian University Books, Troms $\phi$, Norway, 1977.

Bauer, P., K. D. Cole, and G. Lejeune, Field-aligned electric currents and their measurement by the incoherent backscatter technique, Planet. Space Sci., 24, 479-485, 1976. 
Burch, G. H., Operating handbook for digital autocorrelator, contract DASA01-67-C-0019, SRI project 6291-100, SRI Int., Menlo Park, Calif., 1970.

Burch, G. H., Chatanika radar computer interface, operating handbook, NSF grant GA-43190, SRI project 3458, SRI Int., Menlo Park, Calif., 1975.

Carison, H. C., W. E. Gordon, and R. L. Showen, High frequency induced enhancements of the incoherent scatter spectrum at Arecibo, J. Geophys. Res., 77, 1242-1250, 1972.

Chabert, B., P. Dutang, and G. Sanchez, Correlateur multibit à large bande (16 MHz), Note Tech. LETI/MCTE 1045 C.E.A.C.E.N.-G, Lab. d'Electron. et de Technol. de l'Informatique, Grenoble, France, 1974.

Cicerone, R. J., and S. A. Bowhill, Photoelectron fluxes measured at Millstone Hill, Radio Sci., 6, 957-966, 1971.

Dawson, C. H., Atol-The on-line data-collection program for the Chatanika radar facility, operating handbook, NSF grant DES7201644 A02, SRI project 2251, SRI Int., Menlo Park, Calif., 1976.

Evans, J. V., Theory and practice of ionosphere study by Thomson scatter radar, Proc. IEEE, 57, 496-530, 1969.

Farley, D. T., Incoherent scatter correlation function measurements, Radio Sci., 4, 935-953, 1969.

Kamide, Y., and G. Rostoker, The spatial relationship of fieldaligned currents and auroral electrojets to the distribution of nightside auroras, J. Geophys. Res., 82, 5589-5608, 1977.

Kantor, I. J., High frequency induced enhancements of the incoherent scatter spectrum at Arecibo, 2, J. Geophys. Res., 79, 199-208, 1974.

Leadabrand, R. L., M. J. Baron, J. Petriceks, and H. F. Bates, Chatanika, Alaska, auroral-zone incoherent-scatter facility, Radio Sci., 7, 747-756, 1972.

Oran, E. S., V. B. Wickwar, W. Kofman, and A. Newman, Auroral plasma lines: $A$ first comparison of theory and experiment, submitted to $J$. Geophys. Res., 1980.

Papoulis, A., Minimum bias windows for high resolution spectral estimates, IEEE Trans. Inform. Theory, IT-19, 9-12, 1973.

Petriceks, J., Chatanika radar receiver, operating handbook, contract DNA001-74-C-0257, SRI project 3376, SRI Int., Menlo Park, Calif., 1976.

Potemra, T. A., Large-scale characteristics of field-aligned currents determined from the Triad magnetometer experiment, in Dynamical and Chemical Coupling, edited by B. Grandal and J. A. Holtet, pp. 334-352, D. Reidel, Hingham, Mass., 1977.
Rino, C. L., Radar measurement of ionosphere motion in the presence of current-induced spectral asymmetries, Radio Sci., 7, 10491060, 1972.

Rino, C. L., M. J. Baron, G. H. Burch, and O. de la Beaujardiere, A multipulse correlator design for incoherent scatter radar, Radio Sci, 9, 1117-1127, 1974a.

Rino, C. L., M. J. Baron, G. H. Burch, and O. de la Beaujardiere, A multipulse correlator for the Chatanika radar, contract DNA00172-C-0076, SRI project 1703, SRI Int., Menlo Park, Calif., $1974 b$.

Rino, C. L., A. Brekke, and M. J. Baron, High-resolution auroral zone $E$ region neutral wind and current measurements by incoherent scatter radar, J. Geophys. Res., 82, 2295-2304, 1977.

Showen, R. L., The spectral measurements of plasma lines, Radio Sci., 14, 503-508, 1979.

Vidal-Madjar, D., W. Kofman, and G. Lejeune, Mesures de la raie de plasma par diffusion incoherente a Nançay, et premiers resultats morphologiques, Ann. Geophys., 31, 227-234, 1975.

Vondrak, R. R., and M. J. Baron, Radar measurements of the latitudinal variation of auroral ionization, Radio Sci., 11, 939-946, 1976.

Vondrak, R. R., and M. J. Baron, A method of obtaining the energy distribution of auroral electrons from incoherent scatter radar measurements, in Radar Probing of the Auroral Plasma, Proceedings of the EISCAT Summer School, Tromst, Norway, June 5-13, 1975, edited by A. Brekke, pp. 315-330, Scandinavian University Books, Tromsø, Norway, 1977.

Wickwar, V. B., Photoelectrons from the magnetic conjugate point studied by means of the $6300 \mathrm{~A}$ predawn enhancement and the plasma line enhancement, Ph.D. thesis, Rice Univ., Houston, Tex., 1971.

Wickwar, V. B., Plasma lines in the auroral $E$ layer, J. Geophys. Res., $83,5186-5190,1978$.

Wickwar, V. B., M. J. Baron, and R. D. Sears, Auroral energy input from energetic electrons and joule heating at Chatanika, $J$. Geophys. Res., 80, 4364-4367, 1975.

Yngvesson, K. O., and F. W. Perkins, Radar Thomson scatter studies of photoelectrons in the ionosphere and Landau damping, $J$. Geophys. Res., 73, 97-110, 1968.

(Received October 12, 1979;

revised January 3, 1980;

accepted January 23, 1980.) 